\title{
Evaluating a calcium-aware kernel for CT CAC scoring with varying surrounding materials and heart rates: a dynamic phantom study
}

\author{
Niels R. van der Werf ${ }^{1,2}$ (1) Ronald Booij $^{2} \cdot$ Bernhard Schmidt $^{3} \cdot$ Thomas G. Flohr $^{3} \cdot$ Tim Leiner $^{1} \cdot$ Joël J. de Groen ${ }^{2}$. \\ Daniël Bos ${ }^{2,4} \cdot$ Ricardo P. J. Budde ${ }^{2}$. Martin J. Willemink ${ }^{6} \cdot$ Marcel J. W. Greuter $^{5,7}$
}

Received: 25 February 2021 / Revised: 9 April 2021 / Accepted: 17 May 2021 / Published online: 28 May 2021

(C) The Author(s) 2021

\begin{abstract}
Objectives The purpose of this study was twofold. First, the influence of a novel calcium-aware (Ca-aware) computed tomography (CT) reconstruction technique on coronary artery calcium (CAC) scores surrounded by a variety of tissues was assessed. Second, the performance of the Ca-aware reconstruction technique on moving CAC was evaluated with a dynamic phantom. Methods An artificial coronary artery, containing two CAC of equal size and different densities $(196 \pm 3,380 \pm 2$ mg hydroxyapatite $\mathrm{cm}^{-3}$ ), was moved in the center compartment of an anthropomorphic thorax phantom at different heart rates. The center compartment was filled with mixtures, which resembled fat, water, and soft tissue equivalent CT numbers. Raw data was acquired with a routine clinical CAC protocol, at 120 peak kilovolt $(\mathrm{kVp})$. Subsequently, reduced tube voltage $(100 \mathrm{kVp})$ and tin-filtration $(150 \mathrm{Sn} \mathrm{kVp})$ acquisitions were performed. Raw data was reconstructed with a standard and a novel Ca-aware reconstruction technique. Agatston scores of all reconstructions were compared with the reference $(120 \mathrm{kVp})$ and standard reconstruction technique, with relevant deviations defined as $>10 \%$.

Results For all heart rates, Agatston scores for CAC submerged in fat were comparable to the reference, for the reduced-kVp acquisition with $\mathrm{Ca}$-aware reconstruction kernel. For water and soft tissue, medium-density Agatston scores were again comparable to the reference for all heart rates. Low-density Agatston scores showed relevant deviations, up to $15 \%$ and $23 \%$ for water and soft tissue, respectively.

Conclusion CT CAC scoring with varying surrounding materials and heart rates is feasible at patient-specific tube voltages with the novel Ca-aware reconstruction technique.

Key Points

- A dedicated calcium-aware reconstruction kernel results in similar Agatston scores for CAC surrounded by fatty materials regardless of CAC density and heart rate.

- Application of a dedicated calcium-aware reconstruction kernel allows for radiation dose reduction.

- Mass scores determined with CT underestimated physical mass.
\end{abstract}

Keywords Tomography, x-ray computed $\cdot$ Coronary artery disease $\cdot$ Diagnostic imaging $\cdot$ Radiation dosage

$\begin{array}{lll}\text { Abbreviations } & \text { Bpm } & \text { Beats per minute } \\ \text { BAS } \quad \text { Background Agatston score } & \text { Ca-aware } & \text { Calcium aware }\end{array}$

Niels R. van der Werf

n.vanderwerf@erasmusmc.nl

1 Department of Radiology, University Medical Center Utrecht, Utrecht, The Netherlands

2 Department of Radiology \& Nuclear Medicine, Erasmus University Medical Center, Rotterdam, The Netherlands

3 Computed Tomography, Siemens Healthineers, Forchheim, Germany
4 Department of Epidemiology, Erasmus University Medical Center, Rotterdam, The Netherlands

5 Department of Radiology, University of Groningen, University Medical Center Groningen, Groningen, The Netherlands

6 Department of Radiology, Stanford University School of Medicine, Stanford, CA, USA

7 Department of Robotics and Mechatronics, University of Twente, Enschede, The Netherlands 


$\begin{array}{ll}\text { CAC } & \text { Coronary artery calcification } \\ \text { CT } & \text { Computed tomography } \\ \text { FBP } & \text { Filtered back projection } \\ \text { HA } & \text { Hydroxyapatite } \\ \text { HU } & \text { Hounsfield unit } \\ \text { kVp } & \text { Peak tube voltage } \\ \text { mGy } & \text { Milligray } \\ \text { SD } & \text { Standard deviation }\end{array}$

\section{Introduction}

Coronary artery calcifications (CACs), as detected by noncontrast cardiac computed tomography (CT), are a strong predictor for future adverse cardiovascular events [1-3]. With CT, $\mathrm{CAC}$ is traditionally quantified according to the Agatston scoring standard [4]. Other CAC scores, such as the mass score, were developed to decrease shortcomings of the Agatston methodology [5-10]. The number of CT CAC assessments in clinical practice has increased substantially, which in turn increased the cumulative radiation exposure to patients undergoing these exams [11]. In order to standardize CT CAC scoring across different types of CT equipment, a setting of $120 \mathrm{kVp}$ in combination with 3-mm slice thickness is recommended [12]. On the other hand, the most efficient way to reduce radiation dose for CT CAC imaging is to decrease the peak tube voltage $(\mathrm{kVp})$ to values below $120 \mathrm{kVp}$. However, adjusting peak tube voltage will change Agatston scores and has therefore shown to be difficult to implement $[4,10,13]$.

To address this tradeoff, a novel calcium-aware (Ca-aware) reconstruction technique was recently introduced by one of the main CT manufacturers [14]. The aim of this Ca-aware reconstruction kernel is to minimize the previously shown $\mathrm{kVp}$ induced variability of Agatston scores in order to allow for acquisitions at patient-specific lower tube voltages without affecting CAC scores $[15,16]$. This novel reconstruction kernel is optimized for CAC surrounded by fat, as found in vivo where the arteries are embedded in the epicardial fat. In the reconstruction process, bone and calcium are identified after which a voltage-dependent lookup table is used to convert CT numbers to values which correspond to a tube potential of $120 \mathrm{kVp}$. Ideally, this will yield CAC scores obtained at reduced tube voltages that are equivalent to traditional scores that would have been obtained with $120-\mathrm{kVp}$ acquisitions. Changing the resulting tube voltage-dependent Hounsfield unit (HU) values of CAC to their $120-\mathrm{kVp}$ values may enable the use of Agatston calcium scoring methodology independent of tube voltage. For acquisitions with tube voltages below $120 \mathrm{kVp}$, or with added filtration, this may enable decreased radiation dose while maintaining unchanged CAC scores.

An important confounder of CAC scores is residual motion of coronary arteries during image acquisition [17-20]. Motion artifacts can increase or decrease CAC scores, depending on the density of the calcification [20]. While recent studies have assessed the influence of the novel Ca-aware reconstruction technique on both stationary calcifications and in patients, the effect of this reconstruction technique on CAC scores of moving calcifications of different densities at varying heart rates remains unknown [21-23].

Against this background, we formulated the following two aims. Our first aim was to assess the influence of the $\mathrm{Ca}$-aware reconstruction technique on CAC scores of calcifications with different densities surrounded by a variety of patient equivalent tissues. Second, the influence of the Ca-aware reconstruction technique on moving calcifications was assessed with a dynamic anthropomorphic phantom.

\section{Materials and methods}

\section{Phantom}

An anthropomorphic thorax phantom (QRM-thorax, QRM) containing artificial lungs, a spine, and a shell of soft tissue equivalent material was used (Fig. 1). An extension ring of fat

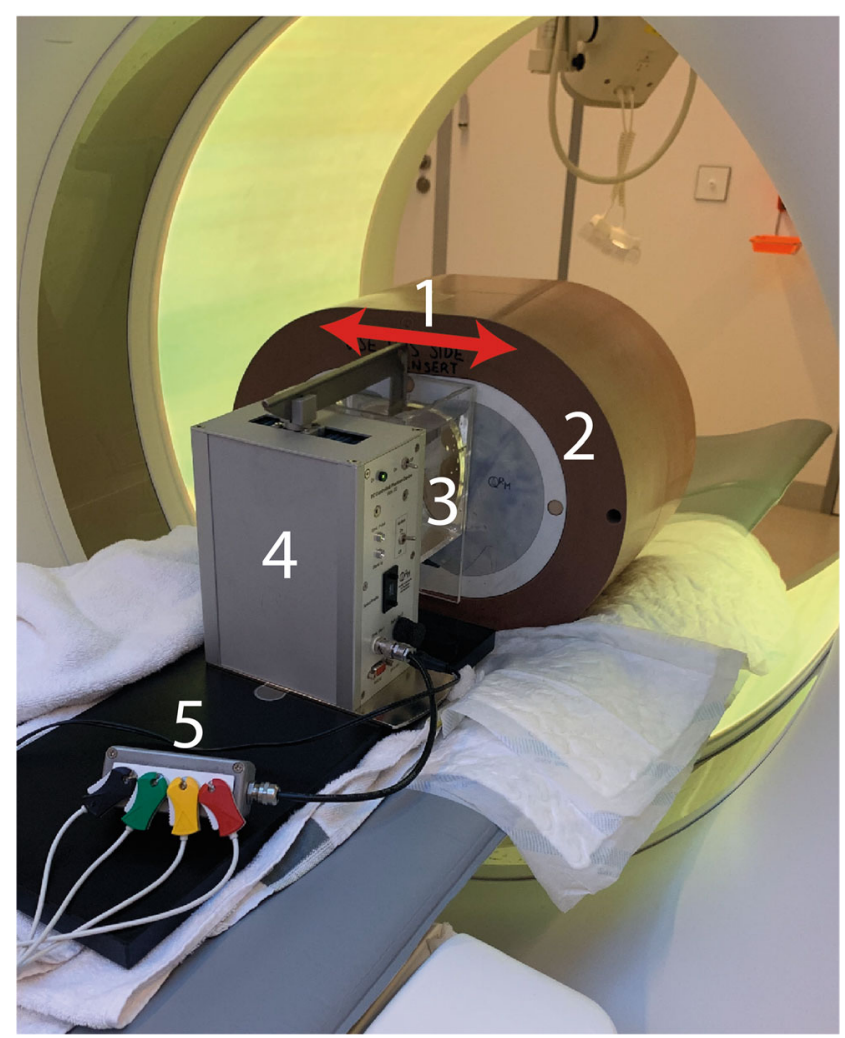

Fig. 1 Overview of phantom setup, with the robotic arm moving a coronary artery along the direction indicated by the red arrow (1), in the anthropomorphic thorax phantom (2), within the fillable compartment (3). Movement is generated with the Sim2D robot (4), which also provides an electrocardiogram output (5) to ensure data acquisition during linear motion of the artificial coronary artery 
equivalent material (QRM-Extension Ring, $Q R M)$ was used to increase the phantom dimensions to $400 \times 300 \mathrm{~mm}$, similar to the dimensions of an averaged-sized patient [15].

A fillable compartment was placed within the cylindrical hole in the center of the thorax phantom, in which an artificial coronary artery was linearly translated at constant velocities of $0,10,20$, and $30 \mathrm{~mm} / \mathrm{s}$ with the use of a robotic arm (Sim2D, QRM). The artificial coronary artery contained both a low- and medium-density calcification of $196 \pm 3$ and $380 \pm 2 \mathrm{mg}$ hydroxyapatite $(\mathrm{HA}) \mathrm{cm}^{-3}$, respectively. Both calcifications were equal in size: $5.0 \pm 0.1 \mathrm{~mm}$ in diameter, with a length of $10.0 \pm$ $0.1 \mathrm{~mm}$. The movement was in a horizontal plane, perpendicular to the scan direction. The velocities of the artificial coronary artery corresponded to the average movement of in vivo coronary arteries during the scan phase at $0,<60,60-75$, and $>75$ beats per minute (bpm) [18]. To ensure that only constant velocities were present during the scan phase, the robotic arm was synchronized with the $\mathrm{CT}$ system during acquisition with the use of the electrocardiography trigger output.

\section{Data acquisition and reconstruction}

Raw data was acquired with a vendor recommended protocol for CT CAC scoring at $120 \mathrm{kVp}$ on a state-of-the-art CT system (SOMATOM Force, Siemens Healthineers) (Table 1). Images were reconstructed with filtered back projection (FBP), using the standard CAC scoring technique (kernel Qr36f), and the Ca-aware reconstruction technique (kernel Sa36). Furthermore, besides the standard 120-kVp acquisition, two other acquisitions were performed. First, data was acquired based on automatic tube voltage selection (CARE $\mathrm{kV}$, Siemens Healthineers) for the water equivalent thickness of the phantom. Second, a dedicated CAC tinfiltration protocol was used. For all protocols, tube current was adjusted according to automatic tube current modulation (CARE Dose4D, Siemens Healthineers) (Table 1). The quality reference was set at $80 \mathrm{mAs} /$ rotation, with the dose optimization slider on position 5 (calcium/bone). Due to a limitation in tube current with automatic tube voltage selection for the used phantom size and $100 \mathrm{SnkVp}$, a tube potential of $150 \mathrm{Sn} \mathrm{kVp}$ was manually selected. To increase sample size and precision, each acquisition was repeated five times for each heart rate. Between each scan, the phantom was manually translated and rotated by approximately $2 \mathrm{~mm}$ and 2 degrees, respectively.

In addition to Agatston scores, we obtained mass scores by acquiring additional images according to each of the three abovementioned protocols with a static cardiac calcification insert that included calcium calibration rods (CCI, QRM). These reconstructions were used to calculate the mass calibration factor for each of our protocols, according to the methodology described by McCollough et al [13].

Table 1 Acquisition and reconstruction parameters for the reference, reduced $\mathrm{kVp}$, and tin-filtration scans

\begin{tabular}{|c|c|c|c|}
\hline Parameter & Reference & Reduced kVp & Tin filtration \\
\hline Acquisition mode & Sequential & Sequential & Sequential \\
\hline Ref. tube voltage $(\mathrm{kVp})$ & 120 & 120 & $100 \mathrm{Sn}$ \\
\hline Ref. tube current product ${ }^{1}$ (mAs/rot) & 80 & 80 & 534 \\
\hline Tube voltage $(\mathrm{kVp})$ & 120 & $100^{2}$ & $150 \mathrm{Sn}^{2}$ \\
\hline Tube current (mAs/rotation) & $100^{3}$ & $148^{3}$ & $136^{3}$ \\
\hline Collimation (mm) & $2 \times 96 \times 0.6$ & $2 \times 96 \times 0.6$ & $2 \times 96 \times 0.6$ \\
\hline Rotation time (s) & 0.25 & 0.25 & 0.25 \\
\hline Temporal resolution (ms) & 66 & 66 & 66 \\
\hline Slice thickness (mm) & 3 & 3 & 3 \\
\hline Slice increment (mm) & $1.5^{4}$ & $1.5^{4}$ & $1.5^{4}$ \\
\hline Kernel & Qr36f/Sa36f $f^{5}$ & Qr36f/Sa36f $f^{5}$ & Qr36f/Sa36f \\
\hline Reconstruction & FBP & FBP & FBP \\
\hline Matrix & $512 \times 512$ & $512 \times 512$ & $512 \times 512$ \\
\hline Field of view (mm) & 220 & 220 & 220 \\
\hline CTDIvol (mGy) & 3.93 & 3.45 & 2.47 \\
\hline
\end{tabular}


The fillable compartment, placed in the anthropomorphic phantom, was used to subsequently acquire data with three materials, adjacent to the artificial coronary artery. These materials resembled fat ( $-100 \mathrm{HU}$, water-ethanol mixture), water (0 HU), and soft tissue (50 HU, water-iodine contrast agent (Iodixanol) mixture). To account for the tube voltage dependency of our mixtures, for each acquisition $(120,100,150 \mathrm{Sn}$ $\mathrm{kVp}$ ), the compartment was filled for that specific tube voltage, as indicated by the flowchart in Fig. 2 for the soft tissue acquisitions. Prior to every acquisition, each mixture was manually stirred to prevent curdling of the liquids. In addition, due to the usage of the robotic arm of the dynamic phantom, the mixture was stirred continuously, except during the $0-\mathrm{mm} /$ $\mathrm{s}$ acquisitions.

\section{Data analysis}

CAC scores were determined from the resulting reconstructed images using a previously validated, in-house developed Python script (Python version 3.7) [21]. A calcium scoring threshold of $130 \mathrm{HU}$ was used for all reference and $\mathrm{Ca}$ aware reconstruction kernel data. For the reduced-kVp acquisition in combination with the standard kernel, a threshold of $147 \mathrm{HU}$ was used, as described previously [16]. As such an adapted threshold was not available for $150 \mathrm{Sn} \mathrm{kVp}$ acquisitions, the regular $130 \mathrm{HU}$ threshold was used. For all acquisitions, mean Agatston and mass scores and standard deviation (SD) were calculated from the five repeated measurements for each combination of heart rate, background material, and acquisition protocol. In addition, an Agatston score was calculated for each reconstruction in a uniform background region-of-interest, without any calcium content. This resulted in a background Agatston score (BAS), which is only larger than zero for high image noise levels, as previously described by Booij et al [21].

For each heart rate, Agatston scores of the reduced-kVp and tin-filtration acquisition were compared to the $120-\mathrm{kVp}$ reference. Differences in Agatston score $\geq 10 \%$ were deemed to be relevant. Resulting mass scores were compared to the physical mass of the calcifications. Again, relevant differences were set at $\geq 10 \%$.

\section{Results}

\section{Background material and radiation dose}

Automatic tube voltage selection, based on the water equivalent thickness of the phantom, resulted in a tube voltage of $100 \mathrm{kVp}$, while $150 \mathrm{Sn} \mathrm{kVp}$ was manually selected. For these protocols, this resulted in a radiation dose of 3.45 and $2.83 \mathrm{mGy}$, respectively. In comparison with the radiation dose of $3.93 \mathrm{mGy}$ for the reference acquisition, this was a reduction of $12 \%$ and $28 \%$ for the reduced-kVp and tin-filtration acquisitions, respectively.

On average, overall velocities and repetitions, background material mean HU ( \pm SD) and image noise values for fat, water, and soft tissue equivalent material, are shown in Table 2. Noise levels from the tin-filtration protocol resulted in $\mathrm{BAS}>0$ for all acquisitions.
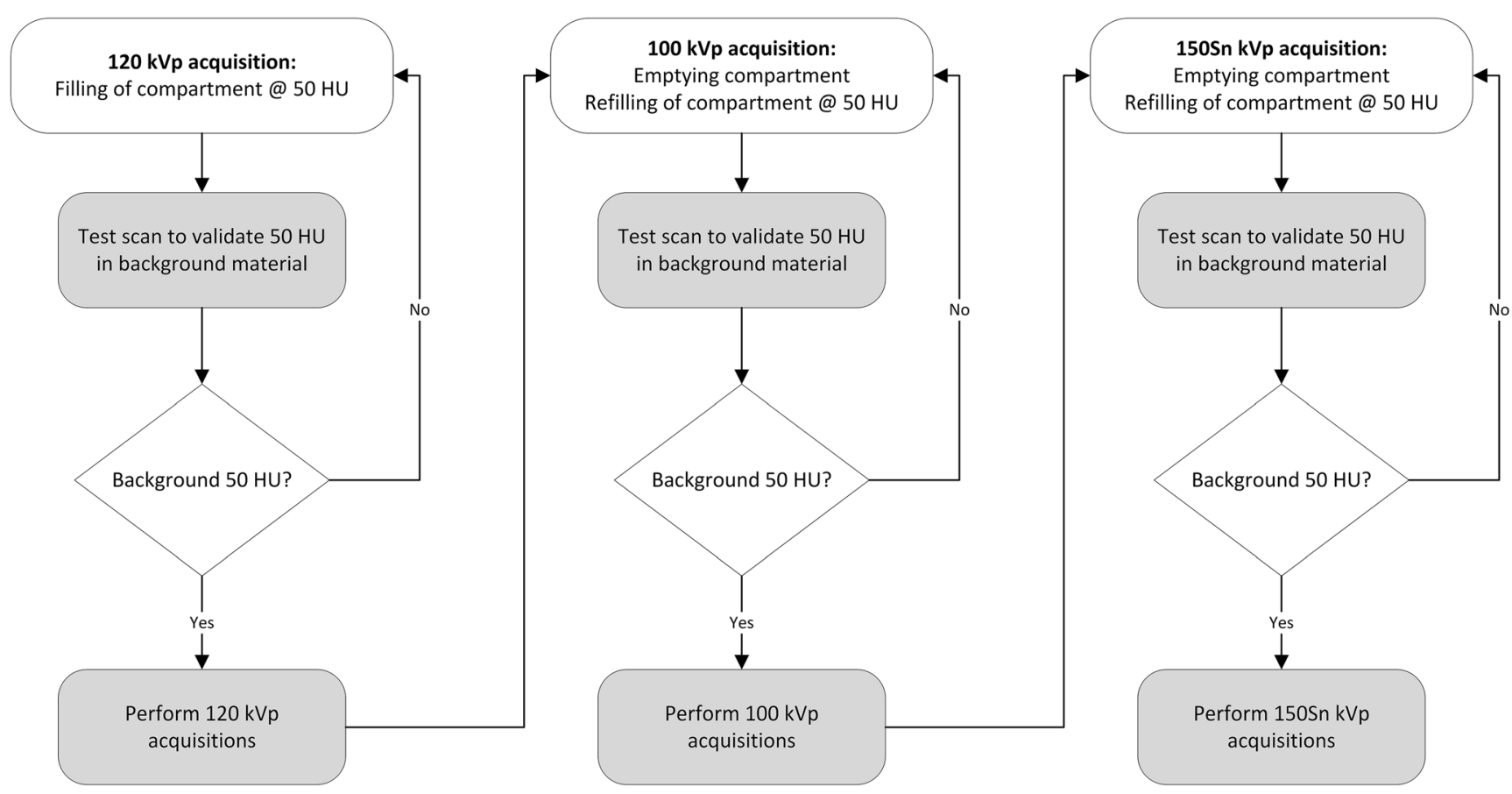

Fig. 2 Flowchart which describes the methodology to account for the tube voltage dependency of the used iodine-water mixture to resemble soft tissue 
Table 2 Background material mean (mean $\pm \mathrm{SD}$ ) and noise (mean $\pm \mathrm{SD}$ ) for all combinations of tube potential, reconstruction kernel, and background material, on average for all used velocities and repetitions

\begin{tabular}{|c|c|c|c|c|c|c|c|}
\hline \multirow[t]{2}{*}{ Tube potential $(\mathrm{kVp})$} & \multirow[t]{2}{*}{ Kernel } & \multicolumn{2}{|l|}{ Fat } & \multicolumn{2}{|l|}{ Water } & \multicolumn{2}{|l|}{ Soft tissue } \\
\hline & & Mean & Noise & Mean & Noise & Mean & Noise \\
\hline \multirow[t]{2}{*}{120} & Standard & $-94.4 \pm 0.2$ & $19.9 \pm 0.7$ & $0.0 \pm 0.4$ & $23.8 \pm 0.3$ & $51.3 \pm 0.4$ & $25.0 \pm 0.4$ \\
\hline & Ca-aware & $-93.8 \pm 0.3$ & $19.9 \pm 0.7$ & $0.8 \pm 0.4$ & $23.8 \pm 0.4$ & $52.3 \pm 0.4$ & $25.0 \pm 0.4$ \\
\hline \multirow[t]{2}{*}{100} & Standard & $-99.0 \pm 0.3$ & $21.0 \pm 1.1$ & $-1.7 \pm 0.3$ & $25.3 \pm 0.4$ & $51.8 \pm 0.3$ & $26.3 \pm 0.6$ \\
\hline & Ca-aware & $-98.5 \pm 0.3$ & $21.2 \pm 1.0$ & $-1.0 \pm 0.3$ & $25.4 \pm 0.4$ & $52.3 \pm 0.3$ & $26.2 \pm 0.5$ \\
\hline \multirow[t]{2}{*}{$150 \mathrm{Sn}$} & Standard & $-84.5 \pm 0.4$ & $23.9 \pm 0.6$ & $2.4 \pm 0.4$ & $27.6 \pm 0.6$ & $49.7 \pm 0.5$ & $28.5 \pm 0.5$ \\
\hline & Ca-aware & $-84.2 \pm 0.3$ & $23.7 \pm 0.3$ & $2.9 \pm 0.4$ & $27.8 \pm 0.6$ & $54.6 \pm 0.6$ & $31.4 \pm 0.7$ \\
\hline
\end{tabular}

\section{Influence of background material on Agatston scores}

Representative images for the highest heart rate are shown in Figs. 3 and 4, for the low- and medium-density calcification, respectively. These figures show a reduced detectability for decreased CAC density and increased surrounding material HU. Agatston scores resulting from the different acquisition and reconstruction protocols are shown in Figs. 5 and 6, again for the low- and medium-density calcification, respectively. From these data, it is clear that the standard reconstruction technique leads to clinically relevant differences for all background materials and heart rates for the low-density CAC.
With the Ca-aware reconstruction technique, Agatston scores are comparable with the reference. For each velocity, deviations from the reference (FBP with standard kernel) were smaller for the medium-density calcification compared to the low-density calcification. Further, almost all tin-filtration acquisitions led to $>10 \%$ differences in comparison with the reference acquisition for all three background materials.

For all heart rates, Agatston scores for the calcifications submerged in fat-like background material were comparable to the reference, for the reduced- $\mathrm{kVp}$ acquisition with $\mathrm{Ca}$-aware reconstruction kernel. For water and soft tissue, medium-density Agatston scores were again comparable to the reference for all
Fig. 3 Overview of representative images of the lowdensity calcifications for different combinations of acquisition and reconstruction parameters, and background material, for $>75$ bpm

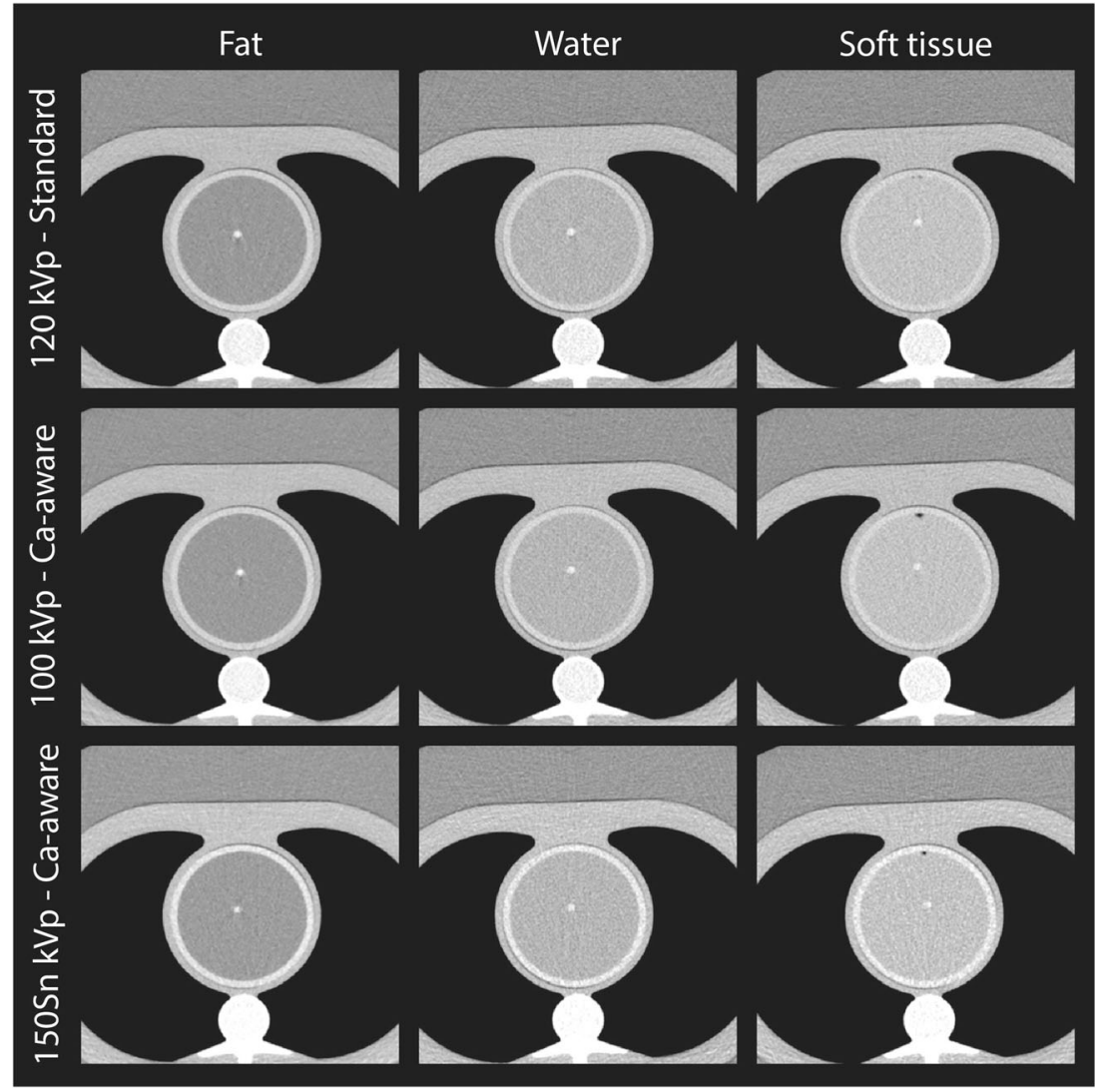


Fig. 4 Overview of representative images of the medium-density calcifications for different combinations of acquisition and reconstruction parameters, and background material, for $>75 \mathrm{bpm}$

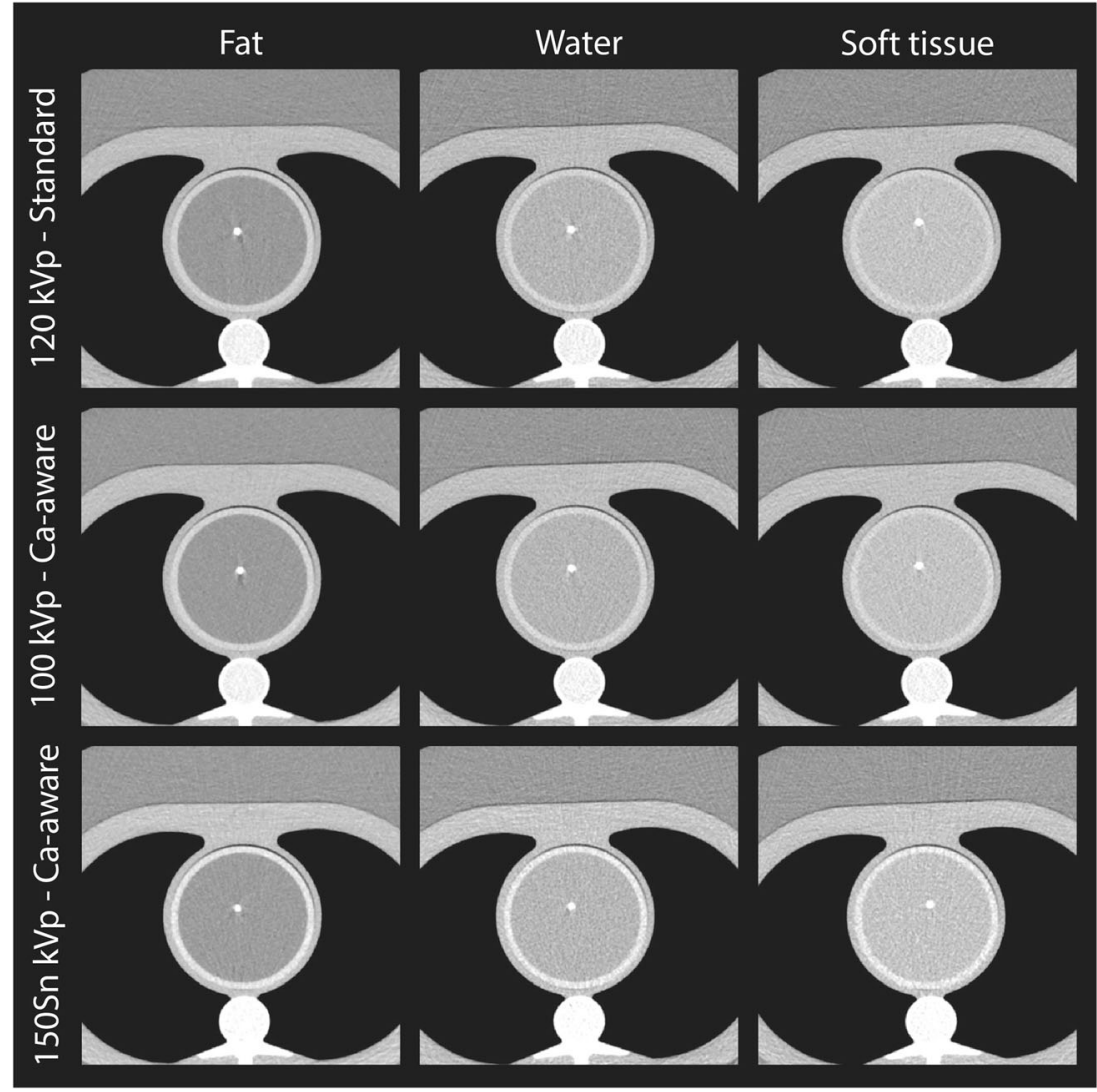

heart rates. However, low-density Agatston scores showed clinically relevant deviations for all heart rates, when the calcification was submerged in water or soft tissue-like material.

\section{Comparison with physical mass}

Physical mass underestimated low-density calcifications by approximately $50 \%$ for all reference acquisition and reconstruction settings (Tables 3 and 4). These underestimations of the physical mass changed to $-50 \%,-41 \%$, and $-34 \%$ for the reduced- $\mathrm{kVp}$ on average for all heart rates, for fat, water, and soft tissue adjacent material, respectively. For the tin-filtration protocol, the underestimation of HU values ranged $-65 \%$, $-53 \%$, and $-31 \%$. Medium-density calcification mass scores underestimated physical mass by $-29 \%,-18 \%$, and $-30 \%$, again on average for all heart rates, for fat, water, and soft tissue circumjacent material, respectively. These values changed to $-31 \%,-20 \%$, and $-9 \%$ for the $\mathrm{kVp}$-reduced protocol, and $-36 \%,-19 \%$, and $-10 \%$ for the tin-filtration protocol.

\section{Discussion}

The main finding of this study is that the Ca-aware reconstruction kernel performs well for a patient-specific tube voltage acquisition protocol (12\% radiation dose reduction), for medium-density CAC, irrespective of CAC adjacent material or heart rate. However, in the presence of low-density CAC, substantial deviations in Agatston scores were observed, when calcifications were surrounded by water (up to 15\%) or soft tissue (up to 22\%) equivalent material, irrespective of heart rate. Furthermore, the tin-filtration protocol also led to substantial deviations in Agatston scores for low-density calcifications, for most combinations of heart rates and surrounding tissue. Furthermore, noise levels for this protocol were high, leading to BAS $>0$. Finally, mass scores as assessed by CT underestimated the true physical mass.

To the best of our knowledge, this study is the first to systematically assess the performance of a novel $\mathrm{Ca}$-aware reconstruction kernel for different CAC surrounding materials, CAC densities, and heart rates. In general, and especially for the low-density calcification, reduced- $\mathrm{kVp}$ acquisitions resulted in increased Agatston scores. This is expected, as the energy dependence of CT numbers of the surrounding material (fat/water/soft tissue) is different from the energy dependence of the CT number of calcium, as previously described by Jakobs et al [24]. Because of the phenomenon, the detectability of calcium is increased, especially at the margins of CAC where voxels might be just below the calcium scoring threshold for $120 \mathrm{kVp}$. In turn, as more CAC is detected, more voxels are taken into account by the $\mathrm{Ca}$-aware reconstruction kernel for its recalculation to $120-\mathrm{kVp} \mathrm{HU}$ values [14]. 
Fig. 5 Deviations in low-density CAC Agatston score from the reference (for each heart rate: 120 $\mathrm{kVp}+$ standard reconstruction kernel) for different heart rates and combinations of tube voltage $(\mathrm{kVp})$ and reconstruction. Results are shown for three background materials: fat ( $-100 \mathrm{HU}$, top), water $(0 \mathrm{HU}$, middle), and soft tissue ( $50 \mathrm{HU}$, bottom). Clinically relevant differences, at $\geq \pm 10 \%$, are indicated with dashed lines

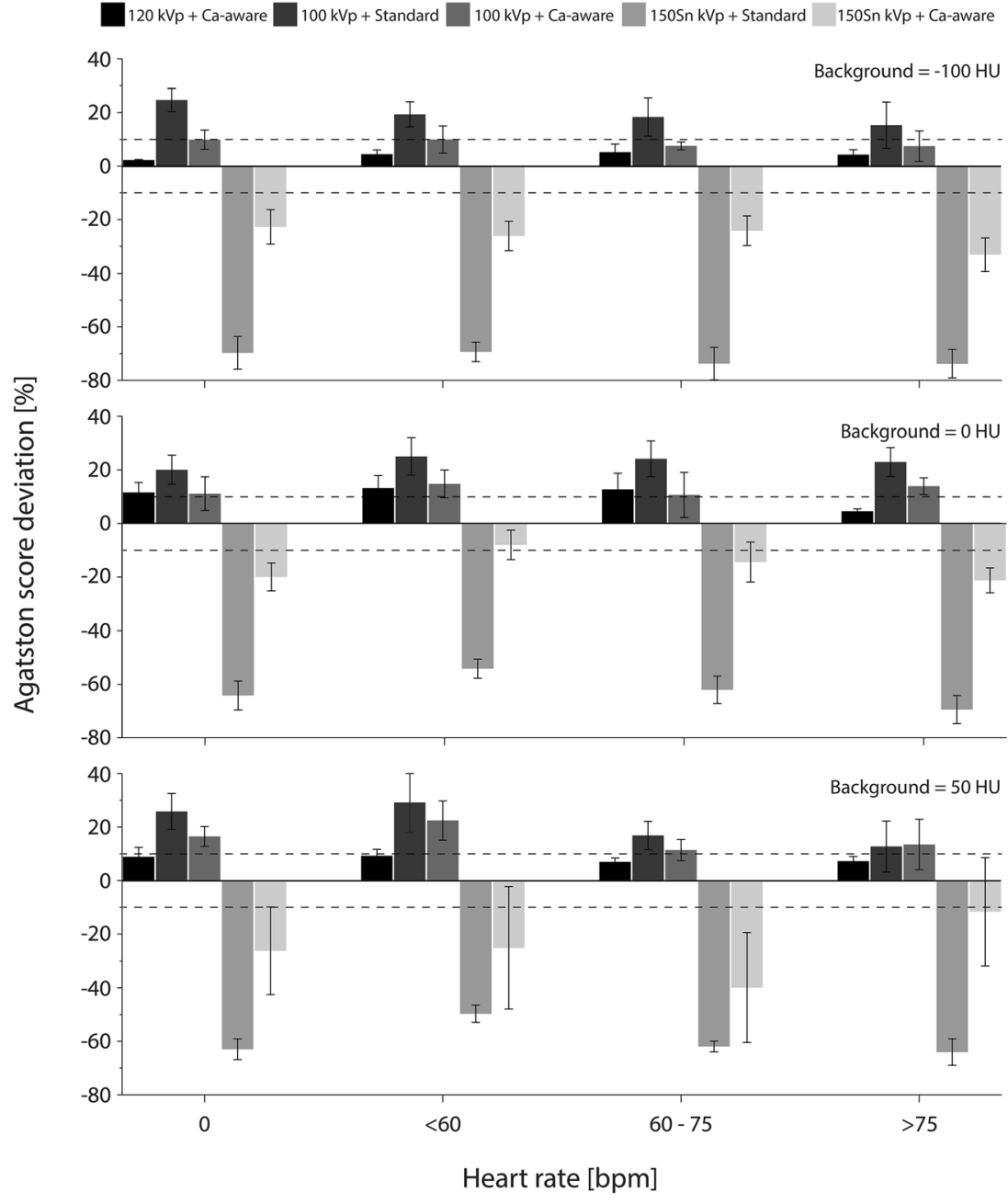

Our results are in line with a phantom study by Booij et al, who demonstrated that the consistency of CT numbers was reduced for low-density CAC when comparing CT numbers from reduced tube voltage acquisitions reconstructed with the $\mathrm{Ca}$-aware kernel, and $\mathrm{CT}$ numbers from routine protocols [21]. In their study, however, a base-material correction factor was provided by the CT manufacturer [14]. This correction factor was necessary to account for the tube voltage dependency of all materials other than water. The usage of this correction factor hampers direct comparison with our results, as this artificial step was necessary due to the nature of the used phantom in their study, which might have influenced resulting Agatston scores.

In addition, two patient studies have been carried out by Vingiani et al $[22,23]$. Both studies showed the feasibility of the $\mathrm{Ca}$-aware reconstruction kernel, in combination with patient-specific tube voltages, where one study considered spectral beam shaping with tin filtration. Comparison of results is hampered by the fact that for their first study, $100 \mathrm{kVp}$ with tin filtration was applied, whereas in our study a tube voltage of $150 \mathrm{kVp}$ with tin filtration was manually selected for the phantom [22]. In the other study by Vingiani et al, forty-three patients were imaged with both $120 \mathrm{kVp}$ and an individualized tube voltage [23]. A high concordance in Agatston scores between both scans was found. Since the density of these CAC and the $\mathrm{HU}$ of the CAC surrounding material are unknown, it is not known if these results are in line or contradictory to our results.

In line with previous studies, we found that CT generally underestimates the physical mass of the low-density calcifications by approximately $50 \%$ for all reference acquisition and reconstruction settings [20].

Our study has some limitations that merit consideration. First, this was an in vitro study, with artificial CAC containing coronary arteries and artificial background material. Nevertheless, the coronary arteries were translated in an anthropomorphic chest phantom at velocities which were observed in in vivo studies [18]. Also, the mass of the calcifications was in the range which is observed in patients [25]. Second, movement of the artificial coronary arteries was only linear and in the horizontal plane, while in vivo complex movements in three dimensions are observed. As the actual scan phase of a CAC scan is only $104 \mathrm{~ms}$, based on the total detector coverage and rotation time, we approximate that the addition of $3 \mathrm{D}$ 
Fig. 6 Deviations in mediumdensity CAC Agatston score from the reference (for each heart rate: $120 \mathrm{kVp}+$ standard reconstruction kernel) for different heart rates and combinations of tube voltage $(\mathrm{kVp})$ and reconstruction kernel. Results are shown for three background materials: fat $(-100 \mathrm{HU}$, top), water (0 HU, middle), and soft tissue (50 $\mathrm{HU}$, bottom). Clinically relevant differences, at $\geq \pm 10 \%$, are indicated with dashed lines
$120 \mathrm{kVp}+\mathrm{Ca}$-aware

$100 \mathrm{kVp}+$ Standard $100 \mathrm{kVp}+\mathrm{Ca}$-aware

$1505 n \mathrm{kVp}+$ Standard $\quad 150 \mathrm{Sn} \mathrm{kVp}+$ Ca-aware

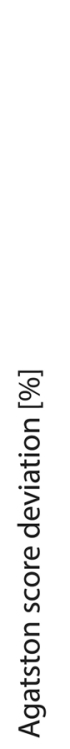

Background $=-100 \mathrm{HU}$
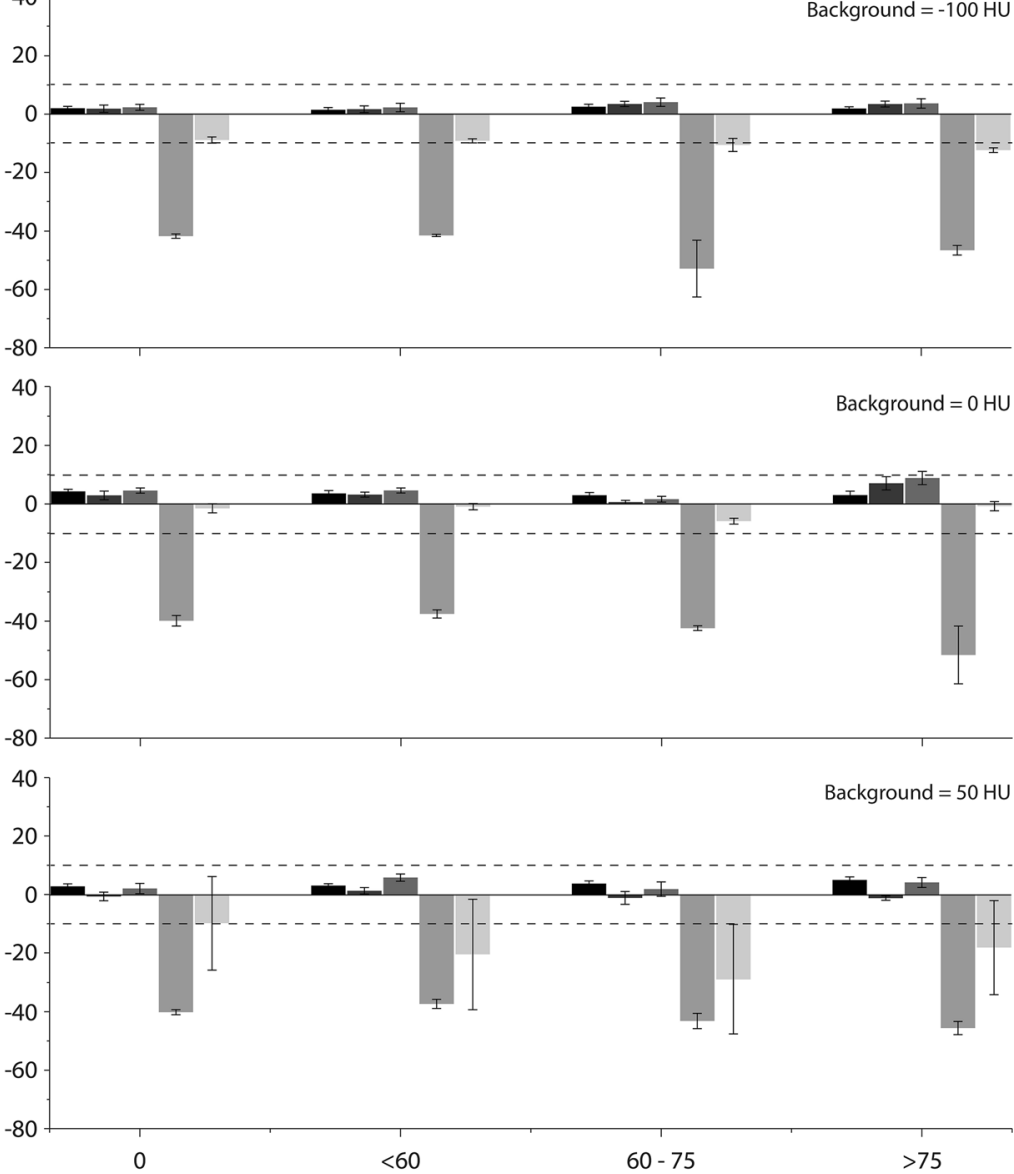

Heart rate [bpm]

Table 3 Percent difference with physical mass of the low-density calcification in mean $\pm \mathrm{SD}$ for all acquisition, reconstruction, background material, and heart rate parameters. To calculate the percentage difference, the numerator was the mass score, and the denominator was the physical mass ( $38 \mathrm{mg}$ )

\begin{tabular}{|c|c|c|c|c|c|c|c|}
\hline \multirow[t]{2}{*}{ Background material } & \multirow[t]{2}{*}{ Heart rate } & \multicolumn{2}{|l|}{$120 \mathrm{kVp}$} & \multicolumn{2}{|l|}{$100 \mathrm{kVp}$} & \multicolumn{2}{|l|}{$150 \mathrm{Sn} \mathrm{kVp}$} \\
\hline & & Standard & Ca-aware & Standard & Ca-aware & Standard & Ca-aware \\
\hline \multirow[t]{4}{*}{ Fat } & 0 & $-47.9 \pm 2.2$ & $-47.8 \pm 2.3$ & $-45.1 \pm 2.0$ & $-46.2 \pm 1.8$ & $-73.7 \pm 5.4$ & $-63.0 \pm 4.1$ \\
\hline & $<60$ & $-48.9 \pm 1.7$ & $-48.8 \pm 1.5$ & $-46.0 \pm 3.0$ & $-47.2 \pm 2.9$ & $-72.8 \pm 2.5$ & $-63.0 \pm 2.7$ \\
\hline & $60-75$ & $-53.5 \pm 0.6$ & $-53.5 \pm 0.6$ & $-51.1 \pm 1.6$ & $-52.6 \pm 1.6$ & $-77.2 \pm 8.8$ & $-64.9 \pm 3.5$ \\
\hline & $>75$ & $-55.8 \pm 1.8$ & $-55.8 \pm 1.8$ & $-54.7 \pm 3.5$ & $-56.0 \pm 3.5$ & $-78.7 \pm 5.3$ & $-69.8 \pm 3.8$ \\
\hline \multirow[t]{4}{*}{ Water } & 0 & $-39.5 \pm 2.9$ & $-38.7 \pm 2.9$ & $-38.0 \pm 2.3$ & $-39.0 \pm 2.3$ & $-62.3 \pm 3.7$ & $-49.9 \pm 2.6$ \\
\hline & $<60$ & $-42.7 \pm 2.3$ & $-42.2 \pm 2.4$ & $-37.7 \pm 1.1$ & $-38.8 \pm 1.0$ & $-59.5 \pm 4.1$ & $-47.5 \pm 3.7$ \\
\hline & $60-75$ & $-41.4 \pm 2.5$ & $-40.7 \pm 2.1$ & $-39.1 \pm 1.6$ & $-40.7 \pm 1.5$ & $-65.3 \pm 7.4$ & $-53.4 \pm 6.7$ \\
\hline & $>75$ & $-47.4 \pm 2.0$ & $-46.8 \pm 1.8$ & $-42.9 \pm 0.7$ & $-44.2 \pm 1.3$ & $-74.3 \pm 8.5$ & $-57.7 \pm 3.0$ \\
\hline \multirow[t]{4}{*}{ Soft tissue } & 0 & $-62.3 \pm 3.7$ & $-49.9 \pm 2.6$ & $-33.5 \pm 2.3$ & $-32.4 \pm 2.0$ & $-28.5 \pm 2.6$ & $-29.8 \pm 2.9$ \\
\hline & $<60$ & $-59.5 \pm 4.1$ & $-47.5 \pm 3.7$ & $-39.3 \pm 6.4$ & $-37.5 \pm 5.9$ & $-26.3 \pm 4.0$ & $-27.6 \pm 4.1$ \\
\hline & $60-75$ & $-74.3 \pm 8.5$ & $-57.7 \pm 3.0$ & $-35.3 \pm 4.5$ & $-33.4 \pm 4.2$ & $-30.5 \pm 3.6$ & $-33.6 \pm 2.6$ \\
\hline & $>75$ & $-74.3 \pm 8.5$ & $-57.7 \pm 3.0$ & $-35.3 \pm 4.5$ & $-33.4 \pm 4.2$ & $-30.5 \pm 3.6$ & $-33.6 \pm 2.6$ \\
\hline
\end{tabular}


Table 4 Percent difference with physical mass of the medium-density calcification in mean \pm SD for all acquisition, reconstruction, background material, and heart rate parameters. To calculate the percentage difference, the numerator was the mass score, and the denominator was the physical mass $(74 \mathrm{mg}$ )

\begin{tabular}{|c|c|c|c|c|c|c|c|}
\hline \multirow[t]{2}{*}{ Background material } & \multirow[t]{2}{*}{ Heart rate } & \multicolumn{2}{|l|}{$120 \mathrm{kVp}$} & \multicolumn{2}{|l|}{$100 \mathrm{kVp}$} & \multicolumn{2}{|l|}{$150 \mathrm{Sn} \mathrm{kVp}$} \\
\hline & & Standard & Ca-aware & Standard & Ca-aware & Standard & Ca-aware \\
\hline \multirow[t]{4}{*}{ Fat } & 0 & $-26.7 \pm 1.2$ & $-27.3 \pm 1.1$ & $-28.2 \pm 0.9$ & $-29.1 \pm 1.0$ & $-36.1 \pm 1.9$ & $-34.0 \pm 1.5$ \\
\hline & $<60$ & $-27.4 \pm 0.4$ & $-28.1 \pm 0.3$ & $-29.4 \pm 1.1$ & $-30.3 \pm 1.0$ & $-36.4 \pm 1.1$ & $-34.1 \pm 1.2$ \\
\hline & $60-75$ & $-29.3 \pm 1.4$ & $-29.9 \pm 1.4$ & $-30.7 \pm 1.3$ & $-31.7 \pm 1.4$ & $-49.9 \pm 19.5$ & $-37.5 \pm 2.6$ \\
\hline & $>75$ & $-34.0 \pm 0.8$ & $-34.5 \pm 0.8$ & $-33.1 \pm 1.6$ & $-34.2 \pm 1.5$ & $-42.8 \pm 2.7$ & $-40.0 \pm 2.2$ \\
\hline \multirow[t]{4}{*}{ Water } & 0 & $-17.4 \pm 1.7$ & $-17.4 \pm 1.7$ & $-18.3 \pm 1.9$ & $-19.1 \pm 2.1$ & $-21.1 \pm 1.3$ & $-16.3 \pm 0.7$ \\
\hline & $<60$ & $-17.7 \pm 1.6$ & $-17.6 \pm 1.7$ & $-18.5 \pm 1.3$ & $-19.2 \pm 1.4$ & $-22.0 \pm 1.0$ & $-18.4 \pm 1.2$ \\
\hline & $60-75$ & $-18.7 \pm 1.0$ & $-18.7 \pm 1.1$ & $-21.2 \pm 1.1$ & $-22.1 \pm 1.1$ & $-26.2 \pm 2.7$ & $-21.6 \pm 2.8$ \\
\hline & $>75$ & $-20.1 \pm 2.1$ & $-20.1 \pm 2.0$ & $-18.8 \pm 1.9$ & $-19.9 \pm 1.9$ & $-38.9 \pm 23.5$ & $-21.1 \pm 2.4$ \\
\hline \multirow[t]{4}{*}{ Soft tissue } & 0 & $-21.1 \pm 1.3$ & $-16.3 \pm 0.7$ & $-10.9 \pm 0.9$ & $-10.6 \pm 0.9$ & $-11.8 \pm 0.8$ & $-12.6 \pm 0.7$ \\
\hline & $<60$ & $-22.0 \pm 1.0$ & $-18.4 \pm 1.2$ & $-12.6 \pm 1.1$ & $-12.2 \pm 1.2$ & $-11.4 \pm 0.5$ & $-12.1 \pm 0.5$ \\
\hline & $60-75$ & $-38.9 \pm 23.5$ & $-21.1 \pm 2.4$ & $-7.0 \pm 1.9$ & $-5.6 \pm 2.1$ & $-5.5 \pm 2.9$ & $-7.3 \pm 2.2$ \\
\hline & $>75$ & $-38.9 \pm 23.5$ & $-21.1 \pm 2.4$ & $-7.0 \pm 1.9$ & $-5.6 \pm 2.1$ & $-5.5 \pm 2.9$ & $-7.3 \pm 2.2$ \\
\hline
\end{tabular}

movement would only result in minor changes in our results. Third, the materials used to simulate in vivo CAC circumjacent tissue are artificial. However, the linear attenuation coefficient of the materials is only used for the Ca-aware reconstruction kernel. The exact chemical composition is therefore irrelevant for the current analysis, as we changed the ratio of our mixtures for each tube voltage to ensure stable background material HU. Fourth, tube current limitations of the dedicated CAC tinfiltration protocol led to the usage of semi-automatic tube voltage selection for our tin-filtration acquisitions and a manual selection of $150 \mathrm{Sn} \mathrm{kVp}$. However, calcium contrast is inherently low for this hardened X-ray spectrum, resulting in reduced CAC detectability and quantification results. Although available to be manually selected by CT radiographers, this $150 \mathrm{Sn}$ protocol is not recommended by the CT manufacturer for CAC scoring for large patients. In addition, a low-dose value was selected for the $150 \mathrm{Sn} \mathrm{kVp}$ protocol which resulted in increased noise levels and may have affected the determination of the Agatston score of the calcifications and may have led to Agatston scores for a non-CAC containing ROI (BAS > 0). This means that the resulting Agatston scores for these acquisitions might be overestimated. However, for all combinations of surrounding material, CAC density, and heart rate, clinically relevant decrease in Agatston score was shown. This decrease should therefore be even larger, when noise levels were lower.

CT CAC scoring with varying surrounding materials and heart rates is feasible at patient-specific tube voltages with the novel Ca-aware reconstruction technique.

Funding The authors state that this work has not received any funding.

\section{Declarations}

Guarantor The scientific guarantor of this publication is Marcel Greuter.

Conflict of interest Two authors (Thomas Flohr and Bernhard Schmidt) of this manuscript declare relationships with the following companies: Siemens Healthineers. The other authors of this manuscript declare no relationships with any companies whose products or services may be related to the subject matter of the article.

Statistics and biometry No complex statistical methods were necessary for this paper.

Informed consent Written informed consent was not required for this study because this is a phantom study.

Ethical approval Institutional Review Board approval was not required because this is a phantom-only study.

\section{Methodology \\ - Prospective \\ - Experimental \\ - Performed at one institution}

Open Access This article is licensed under a Creative Commons Attribution 4.0 International License, which permits use, sharing, adaptation, distribution and reproduction in any medium or format, as long as you give appropriate credit to the original author(s) and the source, provide a link to the Creative Commons licence, and indicate if changes were made. The images or other third party material in this article are included in the article's Creative Commons licence, unless indicated otherwise in a credit line to the material. If material is not included in the article's Creative Commons licence and your intended use is not permitted by statutory regulation or exceeds the permitted use, you will need to obtain permission directly from the copyright holder. To view a copy of this licence, visit http://creativecommons.org/licenses/by/4.0/. 


\section{References}

1. Detrano R, Guerci AD, Carr JJ et al (2008) Coronary calcium as a predictor of coronary events in four racial or ethnic groups. $\mathrm{N} \mathrm{Engl} \mathrm{J}$ Med 358:1336-1345. https://doi.org/10.1056/NEJMoa072100

2. Shaw LJ, Raggi P, Schisterman E et al (2003) Prognostic value of cardiac risk factors and coronary artery calcium screening for allcause mortality. Radiology 228:826-833. https://doi.org/10.1148/ radiol.2283021006

3. Keelan PC, Bielak LF, Ashai K et al (2001) Long-term prognostic value of coronary calcification detected by electron-beam computed tomography in patients undergoing coronary angiography. Circulation 104:412-417. https://doi.org/10.1161/hc2901.093112

4. Agatston AS, Janowitz WR, Hildner FJ et al (1990) Quantification of coronary artery calcium using ultrafast computed tomography. $\mathrm{J}$ Am Coll Cardiol 15:827-832

5. Hong C, Bae KT, Pilgram TK (2003) Coronary artery calcium: accuracy and reproducibility of measurements with multi-detector row CT-assessment of effects of different thresholds and quantification methods. Radiology 227:795-801. https://doi.org/10.1148/ radiol.2273020369

6. Detrano RC, Anderson M, Nelson J et al (2005) Coronary calcium measurements: effect of CT scanner type and calcium measure on rescan reproducibility-MESA study. Radiology 236:477-484. https://doi.org/10.1148/radiol.2362040513

7. Hoffmann U, Siebert U, Bull-Stewart A et al (2006) Evidence for lower variability of coronary artery calcium mineral mass measurements by multi-detector computed tomography in a communitybased cohort-consequences for progression studies. Eur J Radiol 57:396-402. https://doi.org/10.1016/j.ejrad.2005.12.027

8. Rutten A, Isgum I, Prokop M (2008) Coronary calcification: effect of small variation of scan starting position on Agatston, volume, and mass scores. Radiology 246:90-98. https://doi.org/10.1148/ radiol.2461070006

9. Groen JM, Greuter MJ, Schmidt B, Suess C, Vliegenthart R, Oudkerk M (2007) The influence of heart rate, slice thickness, and calcification density on calcium scores using 64-slice multidetector computed tomography: a systematic phantom study. Invest Radiol 42:848-855. https://doi.org/10.1097/RLI. 0b013e318154c549

10. Willemink MJ, van der Werf NR, Nieman K, Greuter MJW, Koweek LM, Fleischmann D (2019) Coronary artery calcium: a technical argument for a new scoring method. J Cardiovasc Comput Tomogr 13:347-352. https://doi.org/10.1016/j.jcct.2018. 10.014

11. Mahabadi AA, Mohlenkamp S, Lehmann N et al (2017) CAC score improves coronary and $\mathrm{CV}$ risk assessment above statin indication by ESC and AHA/ACC primary prevention guidelines. JACC Cardiovasc Imaging 10:143-153. https://doi.org/10.1016/j.jcmg. 2016.03.022

12. Hecht HS, Blaha MJ, Kazerooni EA et al (2018) CAC-DRS: coronary artery calcium data and reporting system. An expert consensus document of the Society of Cardiovascular Computed Tomography (SCCT). J Cardiovasc Comput Tomogr 12:185-191. https://doi. org/10.1016/j.jcct.2018.03.008

13. McCollough $\mathrm{CH}$, Ulzheimer S, Halliburton SS et al (2007) Coronary artery calcium: a multi-institutional, multimanufacturer international standard for quantification at cardiac CT. Radiology 243:527-538. https://doi.org/10.1148/radiol.2432050808

14. Allmendinger T, Hamann A (2019) Agatston score calcium quantification with arbitrary tube voltage [white paper]. In: Siemens Heal. https://www.siemens-healthineers.com/computedtomography/clinical-imaging-solutions/cardiovascular-imaging\# CLINICAL_USE. Accessed Feb 2021

15. Willemink MJ, Abramiuc B, den Harder AM et al (2015) Coronary calcium scores are systematically underestimated at a large chest size: a multivendor phantom study. J Cardiovasc Comput Tomogr 9:415-421. https://doi.org/10.1016/j.jcct.2015.03.010

16. Marwan M, Mettin C, Pflederer T et al (2013) Very low-dose coronary artery calcium scanning with high-pitch spiral acquisition mode: comparison between $120-\mathrm{kV}$ and $100-\mathrm{kV}$ tube voltage protocols. J Cardiovasc Comput Tomogr 7:32-38. https://doi.org/10. 1016/j.jcct.2012.11.004

17. Achenbach S, Ropers D, Holle J et al (2000) In-plane coronary arterial motion velocity: measurement with electron-beam CT. Radiology 216:457-463. https://doi.org/10.1148/radiology.216.2. r00au19457

18. Husmann L, Leschka S, Desbiolles L et al (2007) Coronary artery motion and cardiac phases: dependency on heart rate - implications for CT image reconstruction. Radiology 245:567-576. https://doi. org/10.1148/radiol.2451061791

19. Rutten A, Krul SPJ, Meijs MFL et al (2008) Variability of coronary calcium scores throughout the cardiac cycle: Implications for the appropriate use of electrocardiogram-dose modulation with retrospectively gated computed tomography. Invest Radiol 43:187-194. https://doi.org/10.1097/RLI.0b013e31815cdd56

20. van der Werf NR, Willemink MJ, Willems TP et al (2017) Influence of heart rate on coronary calcium scores: a multimanufacturer phantom study. Int J Cardiovasc Imaging 34:959966. https://doi.org/10.1007/s10554-017-1293-x

21. Booij R, van der Werf NR, Budde RPJ et al (2020) Dose reduction for CT coronary calcium scoring with a calcium-aware image reconstruction technique: a phantom study. Eur Radiol 30:33463355. https://doi.org/10.1007/s00330-020-06709-9

22. Vingiani $\mathrm{V}$, Abadia AF, Schoepf UJ et al (2020) Low-kV coronary artery calcium scoring with tin filtration using a $\mathrm{kV}$-independent reconstruction algorithm. J Cardiovasc Comput Tomogr 14:246250. https://doi.org/10.1016/j.jcct.2019.11.006

23. Vingiani V, Abadia AF, Schoepf UJ et al (2020) Individualized coronary calcium scoring at any tube voltage using a $\mathrm{kV}$ independent reconstruction algorithm. Eur Radiol. https://doi.org/ 10.1007/s00330-020-06951-1

24. Jakobs TF, Wintersperger BJ, Herzog P et al (2003) Ultra-low-dose coronary artery calcium screening using multislice CT with retrospective ECG gating. Eur Radiol 13:1923-1930. https://doi.org/10. 1007/s00330-003-1895-7

25. Hong C, Pilgram TK, Zhu F, Bae KT (2004) Is coronary artery calcium mass related to Agatston score? Acad Radiol 11:286292. https://doi.org/10.1016/s1076-6332(03)00714-1

Publisher's note Springer Nature remains neutral with regard to jurisdictional claims in published maps and institutional affiliations. 